\title{
Search for 1-10 Hz modulations in coronal emission with SECIS during the August 11, 1999 eclipse
}

\author{
P. Rudawy ${ }^{1}$, K. J. H. Phillips ${ }^{2}$, P. T. Gallagher ${ }^{3}$, D. R. Williams ${ }^{4}$, B. Rompolt ${ }^{1}$, and F. P. Keenan ${ }^{5}$ \\ 1 Astronomical Institute of the Wrocław University, ul Kopernika 11, 51-622 Wrocław, Poland \\ 2 National Research Council Senior Research Associate, NASA Goddard Space Flight Center, Greenbelt, MD 20771, USA \\ 3 L-3 Com Analytics Corporation, NASA Goddard Space Flight Center, Greenbelt, MD 20771, USA \\ 4 Mullard Space Science Laboratory, University College London, Holmbury St. Mary, Dorking Surrey RH5 6NT, UK \\ 5 Dept. of Pure and Applied Physics, The Queen's University, Belfast BT7 1NN, N. Ireland, UK
}

Received 15 March 2003 / Accepted 11 December 2003

\begin{abstract}
Results of the search of the periodic changes of the $530.3 \mathrm{~nm}$ line intensity emitted by selected structures of the solar corona in the frequency range $1-10 \mathrm{~Hz}$ are presented. A set of 12728 images of the section of the solar corona extending from near the north pole to the south-west were taken simultaneously in the $530.3 \mathrm{~nm}$ ("green") line and white-light with the Solar Eclipse Coronal Imaging System (SECIS) during the 143-seconds-long totality of the 1999 August 11 solar eclipse observed in Shabla, Bulgaria. The time resolution of the collected data is better than $0.05 \mathrm{~s}$ and the pixel size is approximately 4 arcsec. Using classical Fourier spectral analysis tools, we investigated temporal changes of the local $530.3 \mathrm{~nm}$ coronal line brightness in the frequency range $1-10 \mathrm{~Hz}$ of thousands of points within the field of view. The various photometric and instrumental effects have been extensively considered. We did not find any indisputable, statistically significant evidence of periodicities in any of the investigated points (at significance level $\alpha=0.05$ ).
\end{abstract}

Key words. eclipses - Sun: corona - waves - magnetohydrodynamics (MHD)

\section{Introduction}

The solar corona has a temperature of $1-2 \mathrm{MK}$ and locally much more (Phillips 2000). The detailed heating mechanism is still unknown, but it is certainly related to the magnetic field and is probably due either to dissipation of magnetohydrodynamic (MHD) waves (e.g. Hollweg 1981) or to numerous small-scale magnetic reconnections that result in energy releases of about $10^{23} \mathrm{erg}$, called "nano-flares" (Parker 1988). As evidence of the latter hypothesis, there is evidence of numerous small flare-like phenomena occurring in the quiet solar corona, seen in the ultra-violet (Brueckner \& Bartoe 1993) and in soft X-rays (Shimizu 1995; Koutchmy et al. 1997; Pres \& Phillips 1999; Krucker et al. 1997). However, there is rather contradictory evidence whether the cumulative energy in such events is adequate to heat the corona (Harra et al. 2000; Parnell \& Jupp 2000; Mitra-Kraev \& Benz 2001; Aschwanden \& Charbonneau 2002). In any event, wave heating may still be an important contributor and in certain regions may dominate, e.g. where there are open field lines since at these locations magnetic reconnections are likely to result in plasma acceleration rather than heating.

Send offprint requests to: P. Rudawy, e-mail: rudawy@astro.uni.wroc.pl
Wave heating may result in periodic modulation of the intensity of coronal structures, e.g. in white-light or in the Fe XIV "green" coronal line (wavelength $530.3 \mathrm{~nm}$, emitted at a temperature of about $2 \mathrm{MK}$ ). Theoretical studies of MHD wave heating show that only high frequency $(>0.5 \mathrm{~Hz})$ waves are capable of significant heating (Porter et al. 1994). Such frequencies are therefore higher than what can be observed with spacecraft imaging instruments for which times of about 1-2 $\mathrm{min}$ are required to scan even small areas of the Sun. Hence instrumentation looking for high-frequency intensity modulations must operate from the ground during total eclipses or with coronagraphs. Previous searches with some degree of success have been made by the Williams College (Massachusetts) group observing the green-line corona with photomultiplier tubes (Pasachoff \& Landman 1984; Pasachoff \& Ladd 1987); modulations of $1 \%$ in the coronal intensity with frequencies in the range $0.5-2 \mathrm{~Hz}$ were reported. In more recent eclipses this group has used CCD cameras with fast-frame imaging. Results (Pasachoff et al. 2000) from the 1994 (through thin clouds) and 1998 eclipses (clear skies) did not show any periodicity above a level $>2 \%$ of coronal intensity, but during the August 11, 1999 eclipse (observed in clear skies from Romania) enhanced power was found in the $0.75-1 \mathrm{~Hz}$ range at the 1\% level (Pasachoff et al. 2002). Investigations by other groups (e.g. Cowsik et al. 1999) during eclipses and 


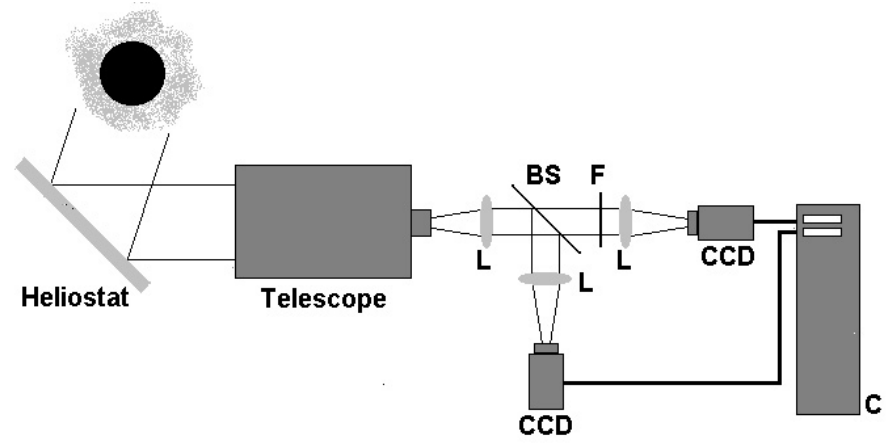

Fig. 1. The optical scheme of the SECIS instrument during the August 11, 1999 total solar eclipse in Shabla, Bulgaria. CCD - camera, C main computer with camera interfaces, $\mathrm{F}-530.3 \mathrm{~nm}$ line filter, BS beam splitter.

with coronagraphs (Koutchmy et al. 1994) have found significant periodicities in the frequency range $0.003-0.14 \mathrm{~Hz}$.

Here we report on analysis of data taken during the August 11, 1999 total eclipse with the Solar Eclipse Coronal Imaging System (SECIS) which is an instrument consisting of a pair of fast-frame CCD cameras with a personal computing system to grab and store the data. In the following, we outline the instrument, observations and necessary corrections to the data. We then describe a Fourier analysis that we carried out in order to search for periodicities in the coronal intensity and the results of the investigations. This work follows reports of periodicities in the same data set using wavelet techniques (Williams et al. 2001, 2002) and we compare our present results with these analyses.

\section{SECIS instrument and observations}

Full instrumental details about SECIS are given by Phillips et al. (2000). It has been used on a number of occasions since the Caribbean total eclipse on February 26, 1998. For the August 11, 1999 eclipse, the setup was as illustrated in Fig. 1. A $250 \mathrm{~mm}$ aperture heliostat (made at the Astronomical Institute, Wrocław University) directed sunlight into a horizontal beam of constant azimuth incident on to a $200 \mathrm{~mm}$, f/10 Meade Schmidt-Cassegrain telescope mounted on an optical bench. Light from the telescope was collimated and then split by a beam splitter into a transmitted beam passing through an interference filter isolating the green-line (bandpass $0.3 \mathrm{~nm}$, central wavelength $530.4 \mathrm{~nm}$; filter manufactured by Barr Associates Inc., USA) then on to one of two cameras, and a reflected beam passing to the second camera with no filter in place. This second, "white-light" camera was thus able to view prominences as well as coronal features which was useful for alignment purposes. Images from this camera also serve as a monitor for atmospheric transmission or any instrumental changes during totality.

The green-line filter's central wavelength has only a slight dependence on ambient temperature, as was confirmed by posteclipse measurements, though the peak transmission $(27 \%$ at $530.4 \mathrm{~nm}$ ) was less than expected. The cameras (manufactured by EEV, UK) have a $512 \times 512$ pixel format (pixel area $15 \mu \mathrm{m} \times 15 \mu \mathrm{m})$. The image data are digitised to 12 bits,

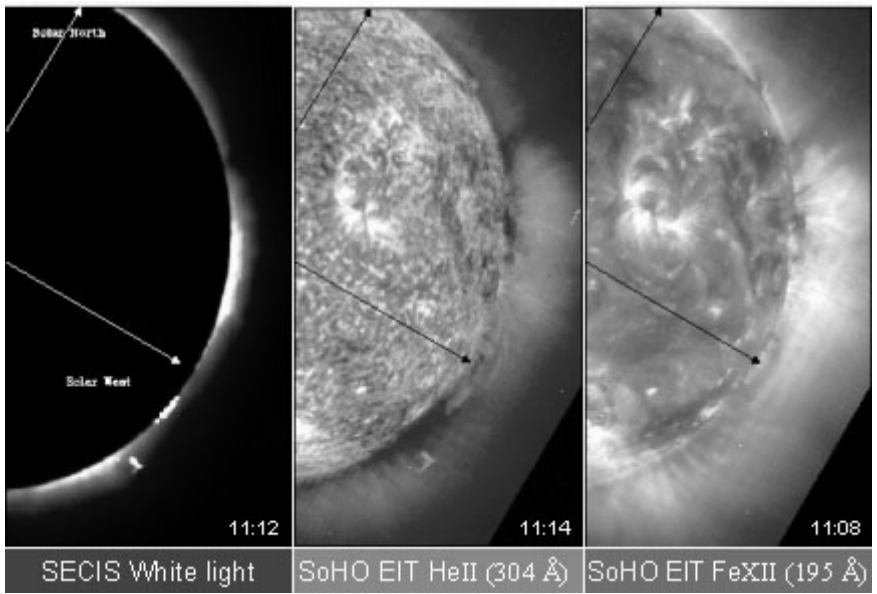

Fig. 2. One of the 6364 images taken with SECIS white-light camera during the 1999 August 11 eclipse (left panel) compared with SOHO EIT image in He II $30.4 \mathrm{~nm}$ line (central panel) and Fe XII $19.5 \mathrm{~nm}$ line (right panel). The prominence used in establishing the image drift is at $\mathrm{S} 21^{\circ}$.

though the least significant bits of data are judged to be noise, so the effective dynamic range of the data is approximately 1000:1. The cameras can work at up to 70 images s $^{-1}$ with exposure times set by the user via an adapted personal computer system (manufactured by Carr Crouch Computer Company, UK) which captures the data streams from the two cameras and stores them on to a $36 \mathrm{~GB}$ disk drive. The spatial scale of the images, determined from pre-eclipse measurements, was 4 arcsec per pixel, giving a field of view of $0.57^{\circ} \times 0.57^{\circ}$, enabling approximately half the visible corona to be viewed at any time.

Using full moon measurements at the Astronomical Institute in Wroclaw, we chose the exposure time to be $20.4 \mathrm{~ms}$ (plus a delay time of $2.1 \mathrm{~ms}$ ), corresponding to a frame rate of $44.4 \mathrm{~s}^{-1}$. At our observing site, a Bulgarian Army establishment at Shabla, $60 \mathrm{~km}$ north of Varna on the Black Sea coast and near the mid-totality line, totality was predicted to be 143 s (Espenak \& Anderson 1997) and weather conditions were expected to be good (Dermendjiev et al. 1998). We started our exposure sequence immediately after the eclipse diamond ring and recorded for $143 \mathrm{~s}$, collecting a total of 12728 images (6364 per camera), or about 6.4 GB of raw data. With ideal weather conditions (cloudless skies and calm air) and a high solar altitude $\left(59^{\circ}\right)$, the image quality matched our expectations, with correct exposure of the white-light images, though with rather low signal-to-noise ratio for some regions of the green-line images. Pre-eclipse $\mathrm{SOHO}$ EIT images enabled the SECIS field of view to be decided before the eclipse. In collaboration with other eclipse observers at Shabla, we selected a region that included the solar west limb from near solar north to the south west. This region (see Fig. 2) included several prominences and coronal features including three bright active regions, two on the north-west limb (NOAA 8651 N25W90, NOAA 8656 N14W86) and one on the south-west limb (NOAA 8661 S14W70). Immediately after totality two sets of flat-field and dark-current images were obtained to enable data corrections to be made. 

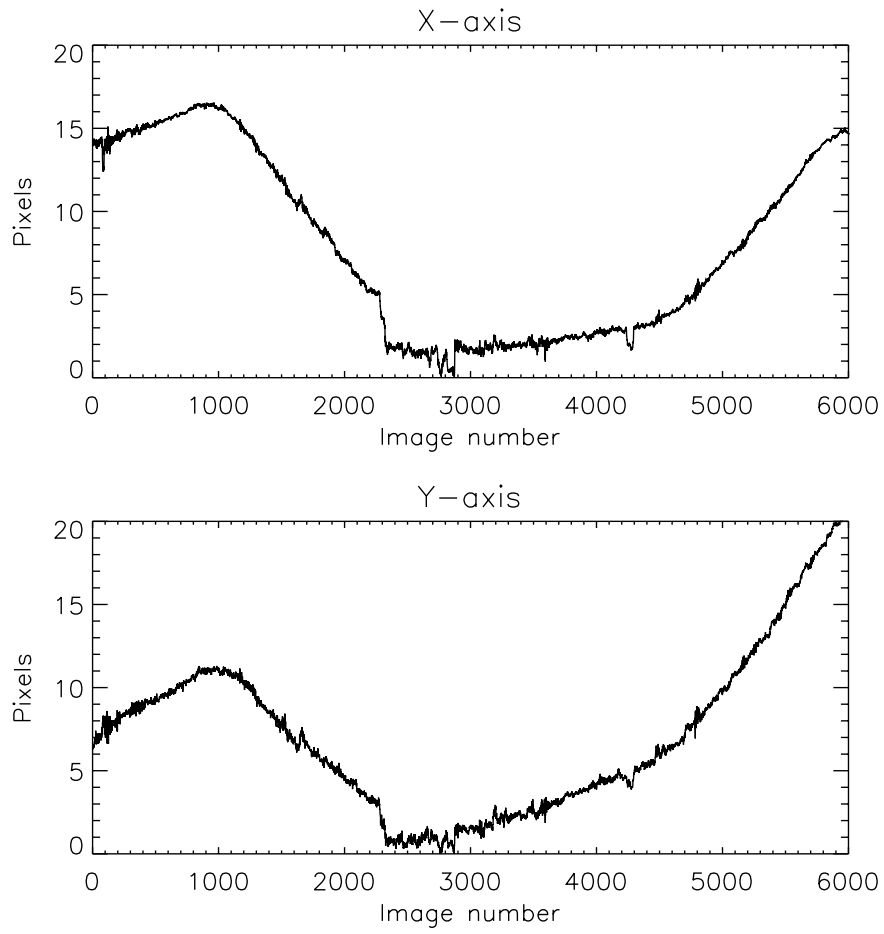

Fig. 3. Temporal changes of the position of the Moon's centre at the SECIS white-light images during the first $134 \mathrm{~s}$ of totality. The coordinates are plotted in an arbitrary reference system. Uncertainties in the plot of corrected positions are too small to be shown here.

\section{Stability and co-alignment of the SECIS images}

Despite pre-eclipse precautions and completely calm conditions, the solar image moved across the detector area of both cameras by up to about 1 arcmin during totality. The motions consist of (1) slow, large-excursion drifts, (2) glitches lasting of order $1 \mathrm{~s}$ and with amplitude approximately 1 pixel (1 px) or less and (3) small, noise-like changes. The cause of the slow drifts and glitches is still under discussion, but it seems possible that dust particles intruded and interfered with the heliostat drive mechanism. (For the June 21, 2001 eclipse, which was also observed by the SECIS instrument, the heliostat drive, previously exposed to the open air, was enclosed by a dust-proof box and the solar image showed no such drifts.) Possibly the noise were caused by atmospheric effects or, to some extent, by the instrument, though the latter is unlikely.

To correct for the image motions, we measured the positions of the Moon's centre and a small, stationary prominence off the south-west limb (S21W) visible in the white-light images (see Fig. 2) for each image. The centre of the Moon was found by least-squares approximation to the visible part of the lunar limb with a section of a circle. Figure 3 shows the temporal changes of the relative positions of the Moon's centre measured from the white-light camera images (unit $=1 \mathrm{px}$ ). They are resolved into $x$ and $y$ coordinates, with $+x$ being upwards in Fig. 2, $+y$ towards the left. Note that these plots do not quite extend to the time of third contact (end of totality) as the final approximately 400 SECIS images in each channel (last $9 \mathrm{~s}$ of totality) were overexposed so preventing an accurate measurement of the Moon's centre position.
The small prominence recorded in SECIS white-light images off the south-west limb (Fig. 2) showed no changes of shape and position during totality, although $\mathrm{SOHO}$ EIT images and eclipse images from Iran (Koutchmy et al. 2001) showed that there were gradual changes over a several-hour interval after 11:11 UT (Portier-Fozzani et al. 2001). We evaluated the position of the prominence's centre of gravity. The temporal changes in the prominence position showed the same drifts, glitches and random noise as those in the Moon's position, but the Moon's position showing in addition a slow, steady motion from approximately west to east, equal to the Moon's proper motion across the Sun.

We note in this context that several previous eclipse experiments have looked for brightness changes of small regions of the solar corona using fibre optics in the focal plane of the telescope coupled to photo-multipliers. These observations rely entirely on the perfect guiding of the heliostat used, which, as we have shown here, cannot be guaranteed and reliably checked for such data after the eclipse is over.

A single-mirror heliostat such as the one we used gives rise to a rotation of the image. This rotation amounts to 2 arcsec $(0.5 \mathrm{px})$ in $30 \mathrm{~s}$ for a feature at the edge of the field of view. To minimise the errors caused by the rotation, we broke the complete sequence of 6364 images per channel into sub-sequences containing 1000 images (i.e. $23 \mathrm{~s}$ long). Here we concentrate on first four of these sub-sequences, starting $0 \mathrm{~s}, 23 \mathrm{~s}, 46 \mathrm{~s}$ and $69 \mathrm{~s}$ after second contact (image numbers from 0 to 3999).

Using measured positions of the Moon and the prominence, we shifted all the images to a common reference system with an absolute accuracy of about one pixel. The shift of each image in a frame of any given sub-sequence was evaluated as a difference between the positions of the prominence or the position of the Moon (taking into account its proper motion) at the reference image and at the actual image. The accuracy of the image pointing obtained, about $1 \mathrm{px}$, is not adequate for reliable analysis of intensity changes in particular parts of the corona. Hence we improved the co-alignment of the images by evaluating the necessary residual shifts of less than 1 px. Such sub-pixel shifts were evaluated using a 2-D correlation of the prominence images at the reference and actual images, both rebinned to 10-times-smaller pixels. Taking into account all the spatial corrections to the SECIS pointing, the time plots of the $x$ and $y$ positions of the Moon's centre are reduced from those showing drifts and short-term glitches to those showing the Moon's steady proper motion of $0.36 \operatorname{arcsec~s}^{-1}$ in an easterly direction, in agreement with predictions (Espenak \& Anderson 1997). Figure 4 shows as an example the original and corrected pointing data for images 1000-1999 in the white-light camera.

The standard deviations of the measured final positions of the Moon from the expected position in the white-light camera images are equal to $0.053 \mathrm{px}$ in $x$ and $0.049 \mathrm{px}$ in $y$ while in the green-line camera images these deviations are equal to $0.098 \mathrm{px}$ and $0.069 \mathrm{px}$. In the four analysed time sets, $99.8 \%$, $99.9 \%, 99.7 \%$ and $99.9 \%$ of the white-light image positions fell within a $3 \sigma$ band while $96.3 \%, 95.9 \%, 94.6 \%$ and $95.2 \%$ of the image positions fell within a $2 \sigma$ range. 

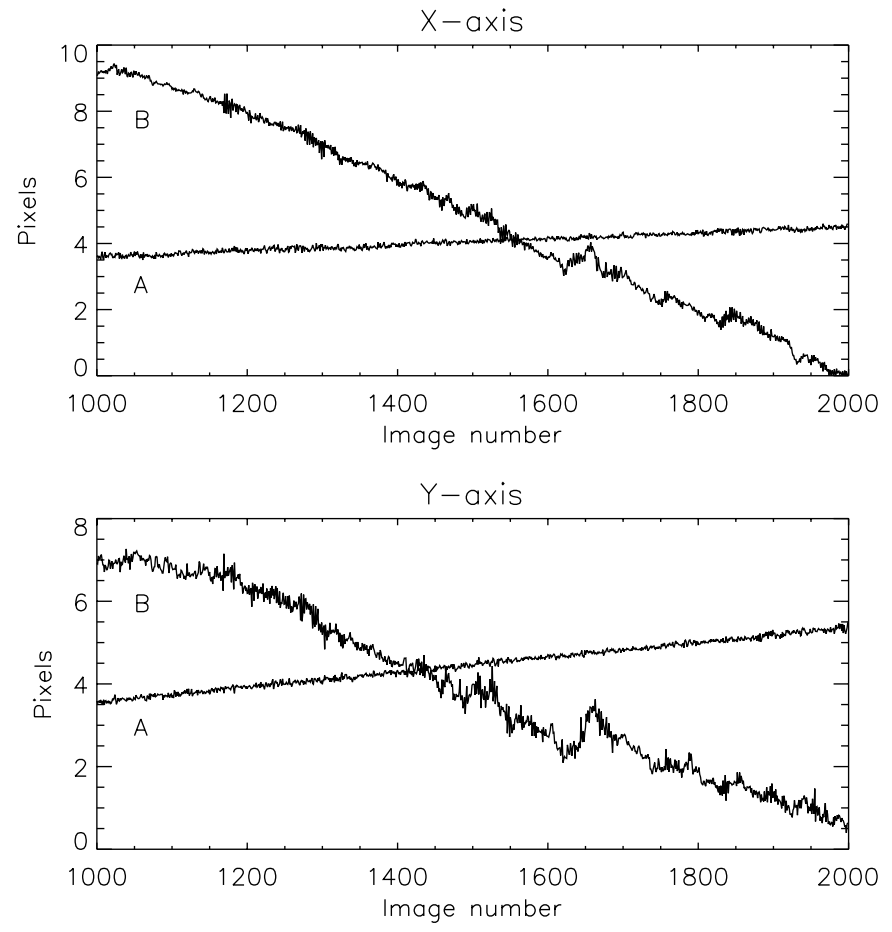

Fig. 4. Temporal changes of the original (labelled B) and corrected (labelled A) position of the Moon's centre for the second set of SECIS white-light images (image numbers 1000-1999). The coordinates are plotted in an arbitrary reference system. Uncertainties in the plot of corrected positions are too small to be shown here.

Since it is possible that residual motions of the image over the CCD detector might give rise to apparent, not real, local intensity changes, we analysed also residual changes of the Moon's centre for each set of data and for both cameras separately. The power spectra of both coordinates did not reveal any statistically important increases (see top two panels of Fig. 5). The temporal variations of the Moon's $x$ and $y$ coordinates are not correlated (the correlation coefficients between them are $0.14,0.04,0.02$, and 0.15 respectively for the four sets of data analysed).

Taking into account the previously evaluated errors, the final accuracy of the co-alignment of the SECIS data, within each individual set of images, is better than $0.1 \mathrm{px}$ in both axes.

We found also that the mean spatial scale of the first 5500 images taken with the white-light camera is equal to $4.05 \pm 0.01 \operatorname{arcsec}$ per pixel $(2977 \mathrm{~km}$ per pixel on the Sun). The mean spatial scale of the first 5500 images captured with the green-line camera is equal to $4.09 \pm 0.02$ arcsec per pixel. These are equal to the spatial scale determined from pre-eclipse terrestrial measurements $(4.1 \pm 0.1$ arcsec per pixel). There are very slight $(<0.1$ arcsec per pixel) variations in the image scales in both channels, uncorrelated in time, which we attribute to inaccuracies in the numerical codes.

\section{Photometric properties of the SECIS data}

Although the CCD cameras used in the SECIS experiment have a $512 \times 512$ pixel format, only the inner $504 \times 504$ pixel region was found to be usable. The central parts of both CCDs have
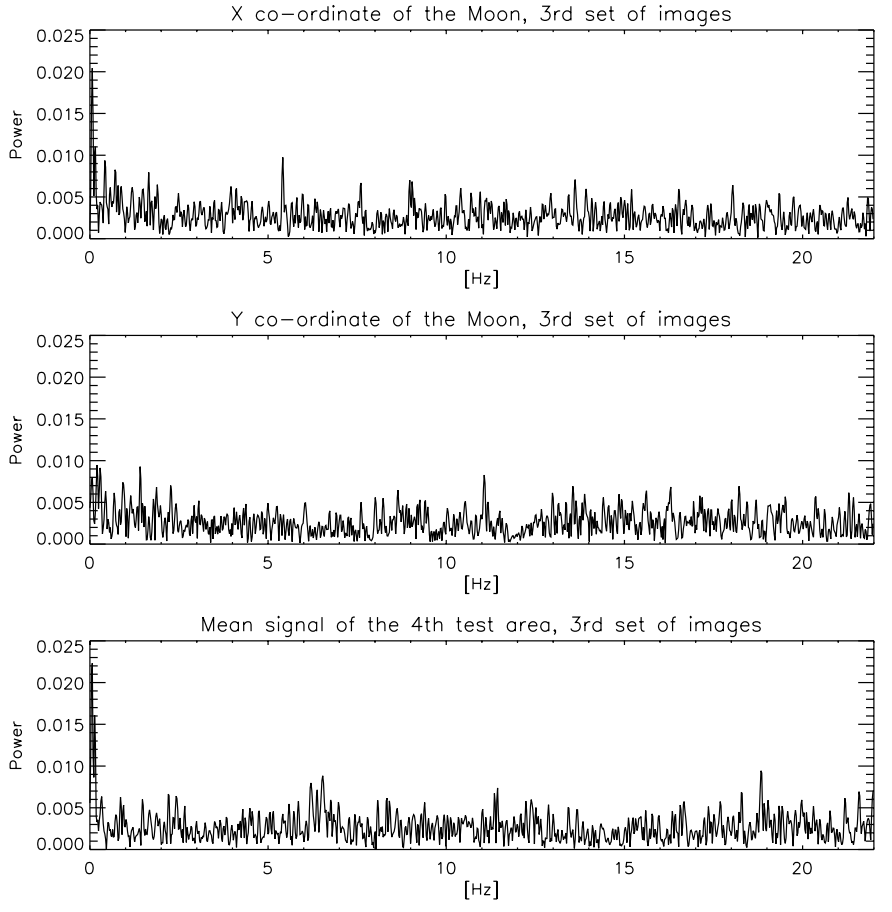

Fig. 5. Top two panels: power spectra of the measured $x$ and $y$ coordinates of the Moon's positions for images of the third data set (46-89 s after second contact) after pointing error corrections. Bottom panel: power spectrum of the mean signal from test area $\mathrm{D}$ taken with the green-line camera.

almost uniform sensitivity but the sensitivity of the leftmost columns (small $x$ values) and upper rows (large $y$ values) differ significantly from the average values. The flat-field frames (taken just after eclipse totality) show periodic patterns and local variations of the sensitivity, apparently imprinted during the manufacturing process and observed in many CCDs. We found also that there was some image vignetting but the most affected parts of the image fortunately lay outside the usable areas. Despite carefully performed standard photometric corrections (subtraction of the dark-current and flat-fielding), for the final photometric analysis of images taken in the green-line we selected only the central part of the images (260 px in $x$, $100 \mathrm{px}$ in $y$, or $1063 \operatorname{arcsec} \times 409 \operatorname{arcsec}$ ); of the $26000 \mathrm{px}$, nearly 17000 px contained detectable coronal emission beyond the lunar limb.

The white-light images were used not only for correction of the pointing errors of the instrument (as already described), but also for monitoring of the temporal changes of the atmospheric transmission and temporal stability of the instrument's photometric properties. Any hidden periodic instability of the photometric properties of the whole instrument or its selected elements (for example one of the cameras) can cause spurious periodic changes of the recorded signal of the coronal green-line. In order to detect such periodicities, we analysed the power spectra of the signals averaged over the selected areas of images taken with both cameras. The power spectra were calculated in a standard way using a Fast Fourier Transform. The mean value of the analysed data was first subtracted, the trend was removed, and the data were multiplied by a bell-shaped 
Hanning's function in order to suppress high frequency components of the Fourier Transform caused by the rapid changes of the signal at both ends of our data sets of limited length (see Press et al. 1986). Random fluctuations of the signal may give rise to local maxima in the power spectrum, which may appear significant when in fact the original signal does not have any periodic component. In order to avoid any misidentification of the detected increases of the calculated power spectra as a real periodicity, we used a significance test due to Fisher (1929) for the largest peak in the power spectrum and an extension to Fisher's test due to Shimsoni (1971) for maxima smaller than the largest one, since according to Shimsoni and authors cited therein Fisher's test tends to be too severe in rejecting peaks as insignificant. We followed Brockwell \& Davis' (1997) discussion of the Fisher test. Fisher's test checks a null hypothesis that the signal $\left\{X_{t}\right\}=\left\{X_{1}, \ldots, X_{n}\right\}$ is white noise only against the alternative hypothesis that the signal contains an added deterministic periodic component of unspecified frequency. The null hypothesis is rejected, if the power spectrum $I\left(\omega_{i}\right),(i=1, \ldots, q)$, contains a value substantially larger than the average value, i.e., with $q=(n-1) / 2$, if

$\xi_{q}:=\left[\max _{1 \leq i \leq q} I\left(\omega_{i}\right)\right] /\left[q^{-1} \sum_{i=1}^{q} I\left(\omega_{i}\right)\right]$

is sufficiently large. To apply the test, one compares a particular value of $\xi_{q}$, say $\xi_{k}$, from data $\left\{X_{t}\right\}$ and computes the probability $P$ that $\xi_{q}$ is greater than or equal to $\xi_{k}$

$P\left(\xi_{q} \geq \xi_{k}\right)=1-\sum_{j=0}^{q}(-1)^{j}\left(\begin{array}{l}q \\ j\end{array}\right)\left(1-j \xi_{k} / q\right)_{+}^{q-1}$

where the subscript + indicates the greater of $\left(1-j \xi_{k} / q\right)$ and zero. If this probability is less than $\alpha$ (the significance level), the null hypothesis is rejected at level $\alpha$ (for details see Brockwell \& Davis 1997).

Using photometrically corrected images taken in whitelight we analysed the temporal variations of the mean signals averaged over four rectangular areas chosen in the solar corona (labelled A, B, C and D, see upper panel of Fig. 6; these areas contain 3654, 4992, 5152 and 4032 pixels respectively), one area inside the image of the lunar disk (labelled E, 11857 pixels) as well as five individual points, chosen for simplicity in the corners of the A, B and C areas (marked by small white squares in Fig. 6). The amplitudes of the averaged signals varied in time by about $0.25 \%$ of their mean values while the signals from individual points varied from $0.5 \%$ to $1 \%$ of their mean values. During the first $2 \mathrm{~s}$ of our observations there was a fast decrease of the mean signals due to the rapidly decreasing scattered photospheric/chromospheric light emitted from the opposite (eastern) edge of the Sun.

Using Fisher's test we did not find any statistically important increases in the power spectra of the averaged white light data. Nevertheless, during further analysis of the coronal greenline emission we decided to apply a more restrictive limitation, ignoring all the oscillations in the green-line emission with frequencies for which the local maximum of the power spectrum of the white-light averaged signal exceeds its linear approximation by more than $2 \sigma$.

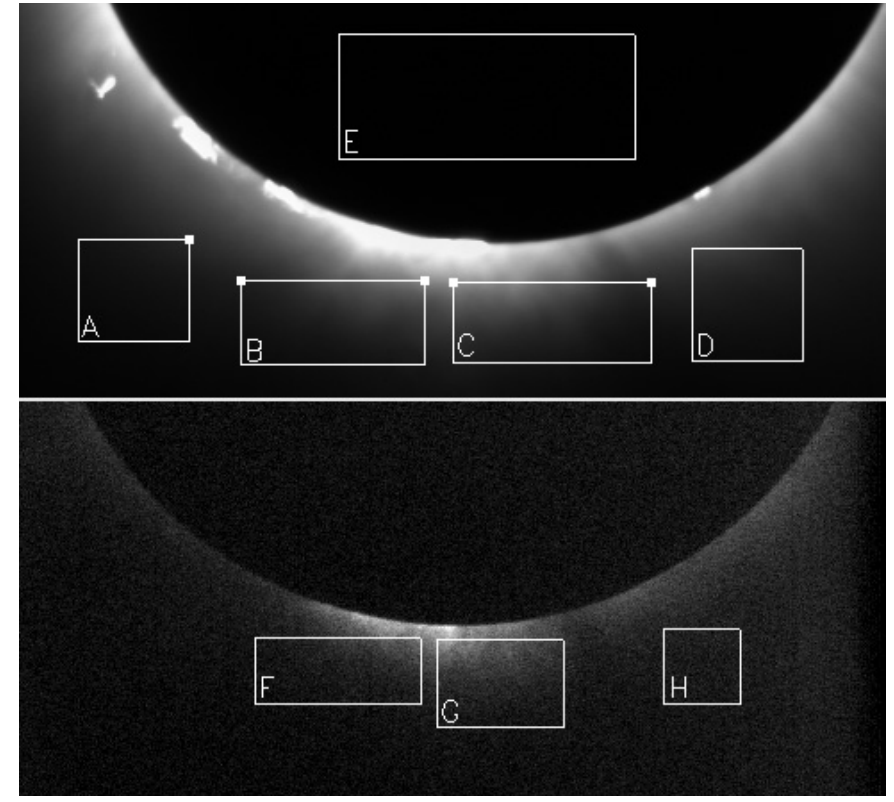

Fig. 6. Upper panel: an image taken with the white-light camera with overplotted five rectangular areas (marked A-E) and five individual point (marked with small white squares) used for investigation of the temporal changes of the atmospheric transmittance and temporal stability of the instrument's photometric properties. Lower panel: an image taken with the green coronal line camera with overplotted areas used for investigation of the photometrical properties of the data taken in green coronal line (see main text for details).

The overall photometric properties of the data taken in the green coronal line were checked by us in the same way as for the white-light data. We analysed the variation of the mean green-line signals averaged over three rectangular areas in the solar corona (encompassing 3512, 3600 and 2322 pixels, labelled F, G and $\mathrm{H}$ respectively, see bottom panel of the Fig. 6). Two areas (labelled F and G) were located inside the bright emission associated with the the two active regions NOAA8656 and NOAA8651 in the green-line images while area $\mathrm{H}$ was outside the bright emission. The signal averaged over each of the areas $\mathrm{F}$ and $\mathrm{G}$ varied in time by less than $2.5 \%$ of its mean value while for area $\mathrm{H}$ the changes were of the order of $5 \%$.

The power spectra of the averaged signals taken in the green-line also did not reveal any statistically important increases (they were calculated and tested in the same way as power spectra of the white-light data). Nevertheless, during the analysis of the brightness variation of the coronal structures observed in this line we also omitted all frequencies for which the power of the oscillations of the mean green-line signal exceeded the linear approximation of the whole power spectrum by more than $2 \sigma$. For example in the third set of data we omitted the following bands between $1 \mathrm{~Hz}$ and $10 \mathrm{~Hz}: 1.3-1.5 \mathrm{~Hz}$, 1.9-2.1 Hz, 5.3-5.5 Hz, 7.5-7.7 Hz and 8.9-9.1 Hz: these ranges correspond to small peaks in the $x$ and $y$ coordinates of the Moon's centre (top two pannels of Fig. 5).

As mentioned before, there was appreciable motion of the images in both channels. It is possible that residual motions after applying the corrections described in Sect. 3 might give rise to intensity changes, particularly in regions of high intensity 
gradient. In order to detect any periodicities of the image positions we analysed the power spectra of the temporal changes of the measured positions of the lunar images in both cameras. The power spectra were calculated and analysed in exactly the same way as previously and they did not reveal any statistically important increases, but, as before, we applied our $2 \sigma$ limitation. An example of the power spectra of the measured $x$ and $y$ coordinates of the lunar positions and power spectrum of the mean signal from test area D of the green-line images, taken between 46 and $89 \mathrm{~s}$ after the second contact (third set of data) is shown in Fig. 5. There is a small peak in the power spectrum of the green-line averaged signal between $6 \mathrm{~Hz}$ and $6.8 \mathrm{~Hz}$ (see Fig. 5, bottom panel), but this peak is not present in power spectra of the image positions (Fig. 5, two upper panels). However, the peak does appear in power spectra of white-light and greenline averaged data in all sets of data. We speculate that it is attributable to an unknown instrumental effect. We have investigated also the correlation coefficients between the temporal changes of the coordinates of the Moon in both cameras and the temporal changes of the averaged signals. The correlations are less than 0.1 , with the exception of the third set of observations, when the correlation coefficients are of the order of 0.2.

\section{Temporal changes of the local brightness in green coronal line}

The analysis of the temporal changes of the brightness of the various coronal structures observed in the green coronal line was made for a area measuring roughly $18 \times 7$ arcmin, including some 17000 pixels having detectable coronal emission. The analysis of the temporal changes of the green coronal line emission in numerous selected points was made using the commercially available statistical software package Statistica (v. 5.5a of StatSoft Inc.) but, to speed the work, we chose pixels having a signal-to-noise ratio greater than 10:1 only (this limited the number of the investigated points to about 6500) and to use personally written routines in Interactive Data Language (IDL, Research Systems, Inc.). For each investigated pixel we calculated power spectra and analysed the temporal variation of the recorded signal during the subsequences of $1000 \mathrm{im}-$ ages each referred to in Sect. 3. The power spectra were calculated using a standard Fourier transformation every $0.01 \mathrm{~Hz}$. Figure 8 shows examples having what appear to be strong peaks. All power spectra showing such peaks were statistically tested using the Fisher test.

The analysis of the local changes of the brightness in the green coronal line was made in several steps. First we calculated the power spectra of the emission for all investigated points in the solar corona and we prepared 2-D maps of the power for each frequency meeting the condition mentioned before that simultaneously the power of the oscillations of the averaged white-light and green-line signals as well as the power of the Moon's position changes did not exceed the linear approximation of the respective whole power spectra by more than $2 \sigma$. It is possible that periodic behaviour can appear in particular solar structures over very short time periods, so we investigated not only the whole data set but also numerous subsets having various durations and arbitrary start times in a
Table 1. Peaks with $>5 \sigma$ and $>4 \sigma$ significance in green line channel pixels

\begin{tabular}{ccccc}
\hline \hline $\begin{array}{c}\text { Set of } \\
\text { images }\end{array}$ & $\begin{array}{c}\text { Detected } \\
\text { periodicity }\end{array}$ & $\begin{array}{c}\text { Start } \\
\text { time }^{1}(\mathrm{~s})\end{array}$ & $\begin{array}{c}\text { Freq. } \\
(\mathrm{Hz})\end{array}$ & $\begin{array}{c}\text { Period of } \\
\text { detection }(\mathrm{s})\end{array}$ \\
\hline \multicolumn{5}{c}{ Points with $5 \sigma$ peaks in the power spectra } \\
\hline 1 & 1 & 0 & 4.4 & $2-20$ \\
2 & 1 & 23 & 7.6 & $23-36$ \\
2 & 2 & 23 & 9.3 & $28-40$ \\
4 & 1 & 69 & 7.9 & $75-86$ \\
\hline \multicolumn{5}{c}{ Points with $4 \sigma$ peaks in the power spectra } \\
\hline 1 & 1 & 0 & 4.0 & $2-20$ \\
1 & 2 & 0 & 4.1 & $2-20$ \\
1 & 3 & 0 & 5.9 & $2-20$ \\
1 & 4 & 0 & 7.1 & $2-20$ \\
2 & 1 & 23 & 3.5 & $25-36$ \\
2 & 2 & 23 & 3.5 & $28-36$ \\
2 & 3 & 23 & 3.8 & $28-36$ \\
2 & 4 & 23 & 4.7 & $28-36$ \\
2 & 5 & 23 & 7.5 & $23-45$ \\
2 & 6 & 23 & 8.2 & $25-36$ \\
2 & 7 & 23 & 9.3 & $25-40$ \\
4 & 1 & 68 & 5.1 & $68-91$ \\
4 & 2 & 68 & 5.7 & $75-86$ \\
4 & 3 & 68 & 6.8 & $75-86$ \\
4 & 4 & 68 & 7.1 & $68-91$ \\
4 & 5 & 68 & 9.9 & $68-91$ \\
4 & 6 & 68 & 9.9 & $75-86$ \\
\hline
\end{tabular}

${ }^{1}$ After 2nd contact.

subsequence. The points with the highest power of the spectrum at given frequency were selected for further detailed investigation. We also selected many points chosen along the most distinguishable coronal structures, traced by the increased intensity of the green-line emission.

We did a careful analysis of the emission in 6390 individual pixels, all covering areas of significant coronal emission in the green line channel. A very small number of these pixels were found to show peaks that at first sight seemed to be of interest. We found 17 points with local maxima of the power spectra greater than $4 \sigma(4,7$ and 6 points in a frame of the first, second and fourth sets of images, respectively) and four points with the local maxima greater than $5 \sigma$. Figures 7 and 8 show the location and the power spectrum of each pixel having $>5 \sigma$ peaks, respectively. The results for all 17 peaks are shown in Table 1, where for individual pixels and time sub-sequences we list the frequency and time-ranges (seconds after eclipse second contact) over which these peaks occurred. However we found not a single one of these peaks satisfied the Fisher test at the $\alpha=0.05$ level (where $\alpha$ is the significance level). We are not therefore claiming that the peaks indicated in Table 1 have any physical significance but rather are most likely the result of random statistical fluctuations. All the remaining pixels 


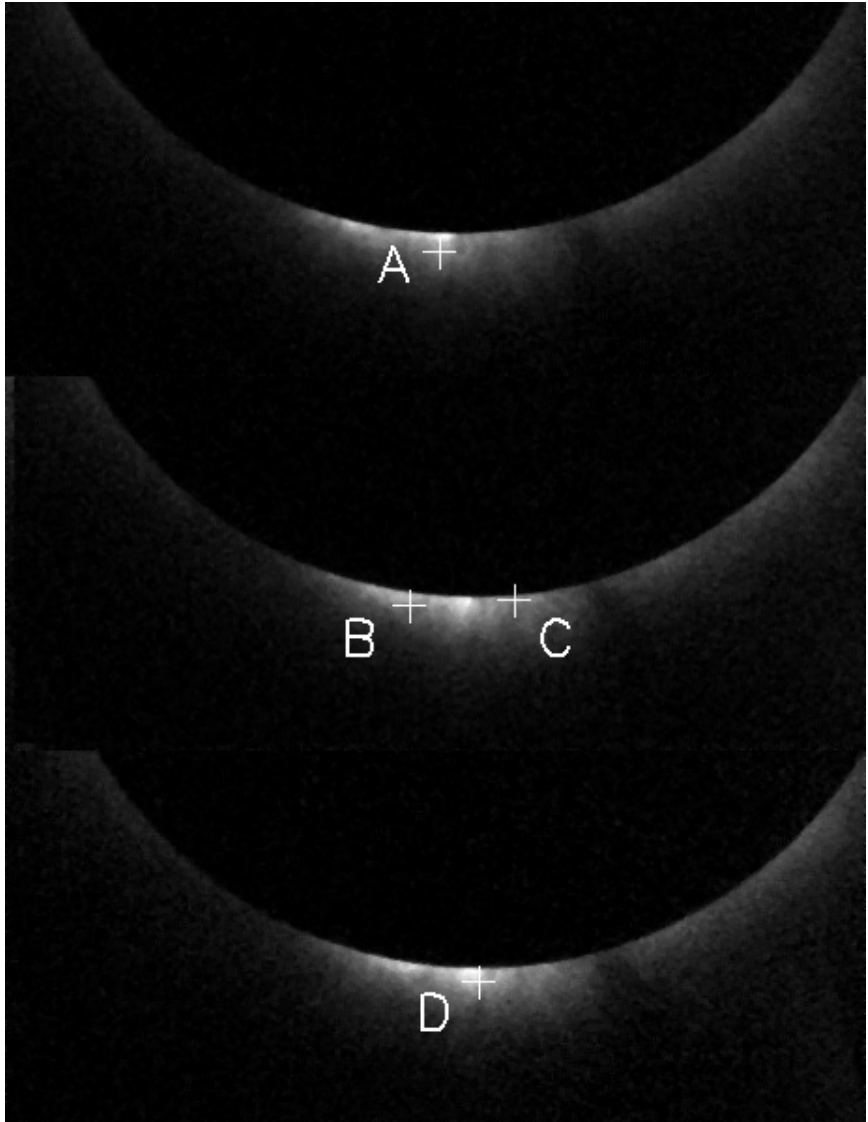

Fig. 7. Location of the points having peaks of the power spectra greater than $5 \sigma$. The points are marked with crosses and labelled by A to D. Upper, middle and lower panels correspond to the first, second and forth set of images, respectively. The spectra of the measured signals are presented in Fig. 8.

showed no peaks in the Fourier power spectra $>4 \sigma$ for any of the four time series.

\section{Discussion of the result and conclusions}

To rule out the possibility that apparent periodicities in the data are due to instrumental or atmospheric effects, we analyzed the effects of various factors, such as the variation of the tracking rate and possible vibrations of the heliostat and the whole instrument, the photometric and other errors associated with the CCDs, possible instabilities of the electronics, variation of the sky transparency and atmospheric seeing and several others.

We are confident that the SECIS data in both channels have been co-aligned within subsequences of 1000 images to 0.1 pixel and that the power spectra of the $x$ and $y$ coordinates of the Moon's centre do not reveal any statistically important peaks. Nevertheless, during the analysis of the brightness variation of the coronal structures observed in the green coronal line we omitted all frequencies for which there is a peak in the power spectrum of the Moon's centre coordinates that exceeds the average level of their power spectra by more than $2 \sigma$. We also tested effects of artificially added instrumental vibrations and found these to be very obvious; their absence in the data indicates such vibrations did not occur.
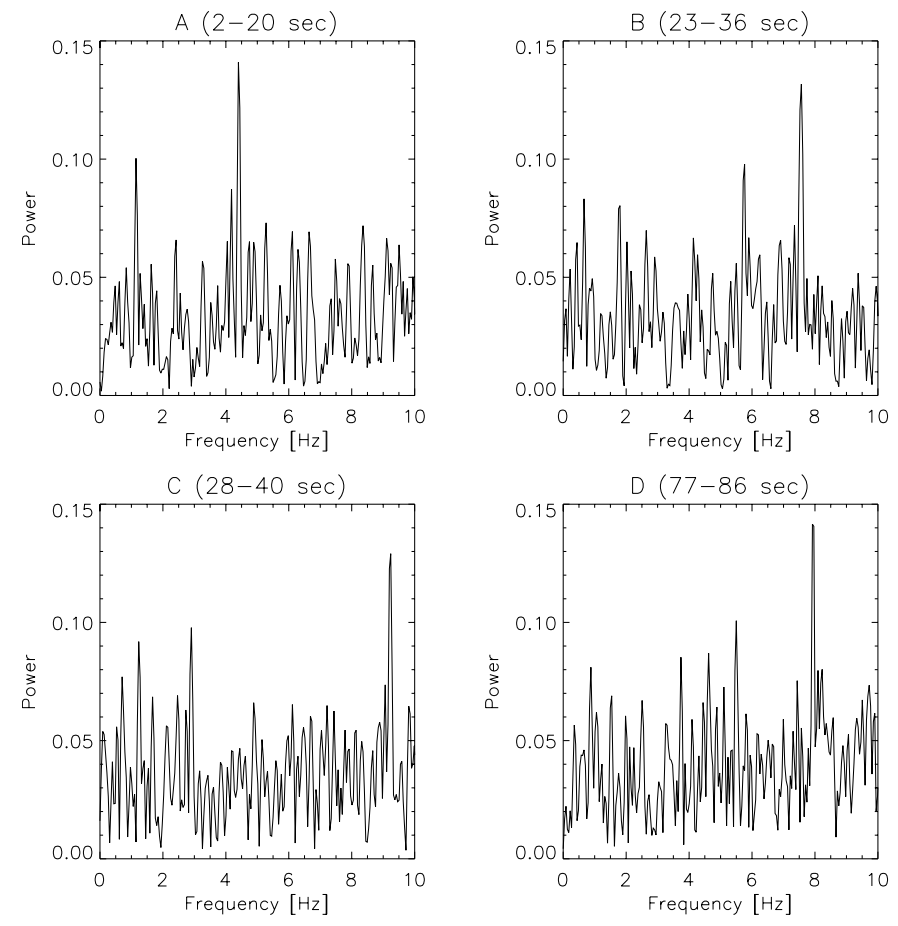

Fig. 8. Power spectra of the $530.3 \mathrm{~nm}$ green coronal line signals recorded for points marked by $\mathrm{A}$ to $\mathrm{D}$ in Fig. 7. The point $\mathrm{D}$ is located close to the coronal loop, where a companion work (see Williams et al. 2001) has found evidence for $0.16 \mathrm{~Hz}$ oscillatory behaviour using a wavelet analysis. Using strict statistical tests we found that local maximum $(7.9 \mathrm{~Hz})$ of the spectrum and numerous similar maxima are statistically unimportant (see main text for details).

The changes of the atmospheric transmission or the change of some selected parameters of the whole instrument (e.g. the temperature of the camera interfaces) can cause periodic changes of the detected green line brightness. These changes can affect whole images. The power spectra of the averaged white-light signals do not reveal any statistically important increases, but nevertheless, during the analysis of the brightness variation of the coronal structures observed in the green coronal line we omitted all frequencies for which the power of the averaged white-light signal oscillations exceeds the linear approximation of the whole power spectrum by more than $2 \sigma$.

We are unable to correct the influence of the atmospheric seeing. The seeing will affect will affect the determination of the Moon's centre position. Unfortunately, the intensity variations and displacements due to the seeing effects usually lie in the frequency interval of interest here $5-10 \mathrm{~Hz}$ (Cowsik et al. 1999). While the seeing effects changed rapidly in frequency and space, they should have a marginal influence on the obtained results.

In summary, our analysis of several thousand pixels in the SECIS green-line images, which included careful correction of several instrumental effects, has not shown any indisputable examples of periodic fluctuations in the data over the $1-10 \mathrm{~Hz}$ frequency range. The coronal emission observed included a bright active region. The absence of significant peaks would argue against the presence of any large oscillatory power in coronal structures that is the signature of MHD wave heating of the corona. It may be that the optical thinness of the 
green-line emission, which allows more than one coronal loop to be seen along a single line of sight, results in a confusion of several possible MHD wave trains so that individual peaks in the power spectra would be difficult to detect. However, there are a few features in our images that are distinguishable as separate loop structures but even here we do not see peaks in the power spectra. A companion work (Williams et al. 2001) has claimed evidence for low-frequency oscillation along one half of a clearly distinguishable loop. This structure was also examined in this work; we found no evidence of significant peaks in the power spectra in the range analyzed here. In the light of the present results the earlier work of Williams et al. will now be investigated.

Although the SECIS results from the 1999 eclipse have high quality and are up to the expectations we had before the eclipse, we have made new observations during an expedition to Zambia to observe the total solar eclipse of June 21, 2001. We used almost the same experimental setup but obtained significantly improved signal-to-noise ratio of the green-line images and much better tracking of the heliostat mirror. The measured tracking errors were about 1 pixel only. The full analysis of the collected data is under way.

Acknowledgements. We thank the Leverhulme Trust (UK) (grant reference F00203/A), Rutherford Appleton Laboratory (Space Science and Technology and Instrumentation Depts.) and the Polish State Committee for Scientific Research (KBN) (grant number is PBZ KBN 054/P03/2001) for financial support. We are grateful to Bulgarian colleagues who arranged the eclipse site at Shabla so well and were particularly helpful in getting our equipment transported in time for the eclipse. We would like to mention specifically Dr Maria Madjarska (now at Armagh Observatory, UK), and particularly the late Prof. Dr Vladimir J. Dermendjiev and the late Prof. Dr Dimitar Mishev (Bulgarian Academy of Sciences, Sofia) for their tireless help before, during and after the eclipse. We also thank the staff of the Bulgarian Army Air Defence Establishment for their help in setting up our base there. We thank Dr Francisco Diego for his help with post-eclipse calibration of the green-line filter. We also thank the several colleagues who gave us technical help, especially Dr Serge Koutchmy, Prof. Jay Pasachoff and Dr Ray Smartt.

\section{References}

Aschwanden, M. J., \& Charbonneau, P. 2002, ApJ, 566, L59

Brockwell, P. J., \& Davis, R. A. 1997, An Introduction to Time Series and Forecasting (New York: Springer), 339
Brueckner, G. E., \& Bartoe, J.-D. F. 1993, ApJ, 272, 329

Cowsik, R., Singh, J., Saxena, A. K., et al. 1999, Sol. Phys., 188, 89

Dermendjiev, V., Mishev, D. I., Tsanev, V. I., \& Popov, G. K. 1998, Advances in Solar Physics Euroconference, ASP Conf. Ser., 155, 403

Espenak, F., \& Anderson, J. 1997, Total Solar Eclipse of 1999 August 11, NASA Ref. Pub. 1398

Harra, L., Gallagher, P. T., \& Phillips, K. J. H. 2000, A\&A, 362, 371

Hollweg, J. 1981, Sol. Phys., 70, 25

Koutchmy, S., Belmahdi, M., Coulter, R. L., et al. 1994, A\&A, 281, 249

Koutchmy, S., Hara, H., Suematsu, Y., et al. 1997, ApJ, 320, L33

Koutchmy, S., Adjabshirizadeh, A., Baudin, F., et al. 2001, Proc. Eclipses and the Solar Corona, ed. P. Durand \& S. Koutchmy, Observations et Travaux, 53, 35

Krucker, S., Benz, A. O., Bastian, T. S., \& Acton, L. W. 1997, ApJ, 488, 499

Mitra-Kraev, U., \& Benz, A. O. 2001, A\&A, 373, 318

Parker, E. N. 1988, ApJ, 330, 474

Parnell, C. E., \& Jupp, P. E. 2000, ApJ, 529, 554

Pasachoff, J. M., \& Landman, D. A. 1984, Sol. Phys., 90, 325

Pasachoff, J. M., \& Livingston, W. 1984, Appl. Optics, 23, 2803

Pasachoff, J. M., \& Ladd, E. F. 1987, Sol. Phys., 109, 365

Pasachoff, J. M., Babcock, B. A., Russell, K. D., McConnochie, T. H., \& Diaz, J. S., 2000, Sol. Phys., 195, 281

Pasachoff, J. M., Babcock, B. A, Russell, K. D., \& Seaton, D. B. 2002, Sol. Phys., 207, 241

Phillips, K. J. H. 2000, Plasma Phys. Control. Fusion, 42, 113

Phillips, K. J. H., Read, P. D., Gallagher, P. T., et al. 2000, Sol. Phys., 193, 259

Porter, L. J, Klimchuk, J. A., \& Sturrock, P. A. 1994, ApJ, 435, 482

Portier-Fozzani, F., Roudier, Y., List, S., \& Roux, E. 2001, Proc. Eclipses and the Solar Corona, ed. P. Durand, \& S. Koutchmy, Observations et Travaux, 53, 66

Pres, P., \& Phillips, K. J. H. 1999, ApJ, 510, L73

Press, W. H., Flannery, B. P., Teukolsky, S. A., \& Vetterling, W. T. 1986, Numerical Recipes (Cambridge Univ. Press), 423

Rusin, V., \& Minarovjech, M. 1994, IAU Symp., 144, 487

Shimizu, T. 1995, Publ. Astron. Soc. Jpn, 47, 251

Singh, J., Cowsik, R., Raveendran, A. V., et al. 1997, Sol. Phys., 170, 235

Williams, D. R., Phillips, K. J. H., Rudawy P., et al. 2001, MNRAS, 326, 428

Williams, D. R., Mathioudakis, M., Gallagher, P. T., et al. 2002, MNRAS, 336, 747

Wood, B. E., Karovska, M., Cook, J. W., et al. 1998, ApJ, 505, 432 